Variations sur la lettre, le mètre et la mesure :

Shakespeare

\title{
Le livre et la blessure
}

Yves Peyré

\section{(2) OpenEdition \\ 1 Journals}

\section{Édition électronique}

URL : http://journals.openedition.org/shakespeare/976

DOI : $10.4000 /$ shakespeare. 976

ISSN : 2271-6424

Éditeur

Société Française Shakespeare

\section{Édition imprimée}

Date de publication : 1 novembre 1996

Pagination : 99-110

Référence électronique

Yves Peyré, "Le livre et la blessure », Actes des congrès de la Société française Shakespeare [En ligne],

14 | 1996, mis en ligne le 01 janvier 2007, consulté le 25 avril 2019. URL : http://

journals.openedition.org/shakespeare/976; DOI : 10.4000/shakespeare.976 


\section{Variations sur la lettre,}

le mètre et la mesure

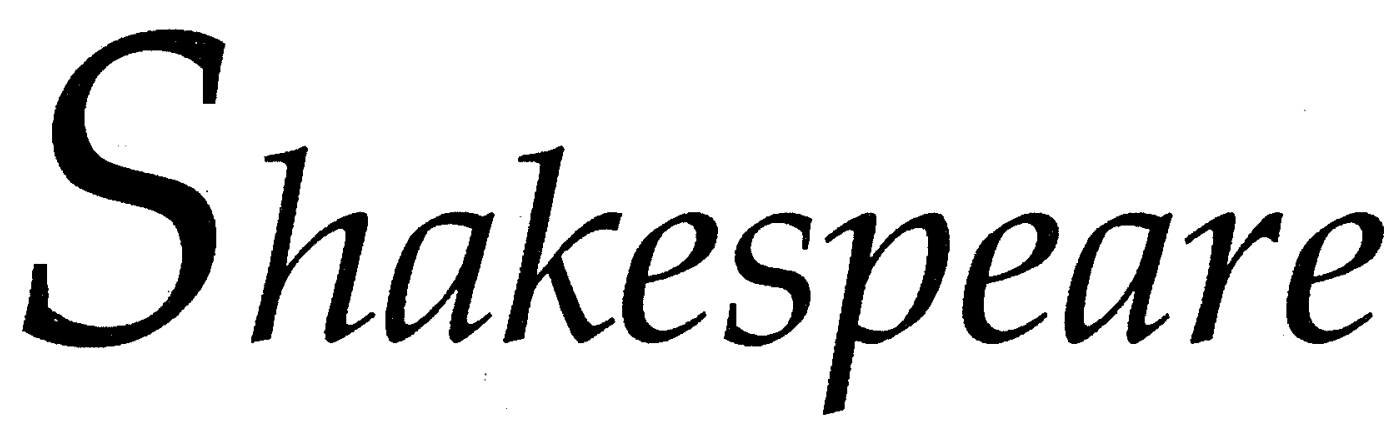

textes présentés par Dominique Goy-Blanquet

Directeur de la publication : Richard Marienstras 


\section{Le livre et la blessure}

L'importance et la variété de la métaphore du livre dans l'œuvre de Shakespeare, déjà remarquée par Goethe ${ }^{1}$, a fait l'objet d'une étude d'Ernst Robert Curtius qui, dans La Littérature européenne et le moyen-âge latin (ch. 16.9, pp. 408-20), pose de précieux jalons dans l'immensité de ce champ métaphorique. C'est la carte d'un secteur beaucoup plus restreint que tente de dresser la présente étude, en se limitant à certaines des images qui métamorphosent le vivant, c'est-à-dire, le plus souvent, l'homme, en page écrite ou lue. Loin d'être propre à Shakespeare, le motif se déploie, de Thomas Kyd à John Ford, dans l'ensemble du théâtre anglais de la Renaissance, dont il constitue un lieu commun constamment reformulé selon de nouvelles modalités.

Dans la première partie de Tamburlaine, les caractères de la valeur martiale sont gravés sur le front de Theridamas (I.2.168), tandis que sur le visage de Xenocrate, la Beauté "comments volumes with her ivory pen" (V.1.145). Ce que Piero Camporesi nomme "le travail étonnant du corps humain", "imprimant une surface et une forme à l'invisible, traçant l'impalpable essence de l'âme sur la peau, gravant sur la façade les signes du profond ${ }^{2}$ ", produit un livre où se lit l'expérience intérieure de l'individu, non seulement le courage ou la perfection, mais aussi les blessures qu'infligent le temps, le chagrin ou le péché. Dans The Comedy of Errors les tourments ont inscrit sur le visage d'Egeon d'étranges déformations (V.1.298300). Dans David and Bethsabe, Semei condamne "The sinnes of David printed in his browes" (1314). Richard II trouve en son propre visage le livre de la connaissance de soi, "the very book indeed / Where all my sins are writ, and that's myself" (IV.1.273-75). C'est aussi le livre de la conscience, ailleurs constamment malmené, repoussé par Bianca, dans Women Beware Women (IV.3.48-51), invoqué, non sans impudence, comme "livre de vérité" par De Flores dans The Changeling (III.4.134), ou comme "registre noir" par Bosola dans The Duchess of Malfi (IV.2.384-86). Dans The Maid's Tragedy, bien que les transgressions morales ne marquent plus le corps de leurs blessures, Evadne se dit prête à les arborer sur son front (IV.1.33-4); pourtant, son corps entier ne pourrait en contenir la longue liste; "Thy body is too little for the story" (IV.1.35), persifle Melantius, qui menace de la transformer en récit de sa propre honte : après l'avoir tuée, il la laisserait nue, "That on thy branded flesh the world may read / Thy black shame and my justice" (IV.1.112-13). Profondément imprimée dans le corps, la flétrissure est un mode d'écriture qui, contrairement à la plupart des autres, reste indélébile.

Si l'écrit est dépositaire de la mémoire des hommes, la mémoire est ellemême texte ${ }^{3}$, que l'on voudrait parfois effaçable, parfois éternel; après avoir gommé de "the table of my memory" tout ce qui l'encombrait, Hamlet s'engage à ne garder que l'injonction du spectre "Within the book and volume of my brain" (I.5.98-103); 
mais il s'agit là d'un texte qui, lors du déroulement de la pièce, semble être constamment perdu de vue, et a toujours besoin d'être reformulé. Macbeth a beau promettre à Ross et Angus :

\section{Kind gentlemen, your pains \\ Are register'd where every day I turn \\ The leaf to read them}

bientôt, l'écriture s'abîme et s'efface, le livre se referme. A partir de ces blessures d'un texte ou trop instable ou trop permanent, les relations dramatiques s'organisent en termes de lecture ou d'écriture : on donne son corps et son être à lire ou on les ferme au regard; on cherche à déchiffrer le corps ou le cœur d'autrui, ou à les marquer de l'empreinte de son écriture.

La valeur hérö̈que s'offre à la lecture sur le corps martial fièrement exhibé. Dans Antony and Cleopatra, le soldat Scarus, dans le nom même duquel s'inscrivent ses blessures, arbore "a wound here that was like a T, / But now 'tis made an H" (IV.7.6-7). Octave, lui, met l'accent sur le témoignage des torts subis, "The record of what injuries you did us, / [...] written in our flesh" (V.2.118-20). Mais archivée sur la peau, la mémoire demeure éphémère; dans The Raging Turk de Thomas Goffe, la Paix endort les citoyens et "wipeth out their scarres / Writ on their bosoms by the hand of warres" (1349-50); un excès de fierté produit sinon un effacement du moins une occultation dans Coriolanus lorsque le protagoniste refuse de dévoiler à la plèbe ces inscriptions corporelles ${ }^{4}$. Il y a dans la lecture une prise de possession du corps de l'autre. C'est pourquoi, se sentant menacé dans son intégrité, Coriolan refuse d'ouvrir le livre. Dans Troilus and Cressida Hector trouve un autre refuge en opposant d'indéchiffrables profondeurs (IV.5.238-39) au regard qu'Achille promène méprisamment sur son corps pour y trouver l'endroit où il le tuera :

\section{I have with exact view perus'd thee, Hector, And quoted joint by joint.}

(IV.5.231-32)

La menace d'Achille peut paraître équivoque, dans une pièce qui enchevêtre et confond le registre guerrier et le registre érotique, dans la mesure où la lecture du corps est fréquemment une activité amoureuse, souvent sensuelle, comme le rappelle Ulysse, selon qui l'apparence et les moindres mouvements de Cressida semblent des invites qui "wide unclasp the tables of their thoughts / To every ticklish reader" (IV.5.60-61).

Sans doute l'absorption dans le texte que constitue le regard ${ }^{5}$ est censée traduire un sentiment intense; dans Dido Queen of Carthage, dès qu'elle s'enflamme pour Enée, la reine s'écrie "His looks shall be my only library" (III.1.89); mais si les yeux d'Helena, dans A Midsummer Night's Dream, offrent à Lysander "Love's stories, written in love's richest book" (II.2.121), le compliment porte à faux, 
comme l'évocation élaborée du visage de Paris dans Romeo and Juliet (I.3.81-92). Le corps humain est textualisé - ou peut-on dire littéralisé ? - lorsque la nourrice donne un sens impudique à la lettre R (II.4.220), ou lorsque Malvolio croit reconnaître l'écriture d'Olivia, "her very C's, her U's, and her T's, and thus makes she her great P's" (Twelfth Night, II.5.88-9). Dans Monsieur d'Olive, après avoir tiré parti des potentialités érotiques de la ponctuation, comme l'avait déjà fait Barnabe Barnes dans un poème de Parthenophil et Parthenophe (élégie 2$)^{6}$, Roderigue prétend qu'il n'y a jamais trop de $\mathrm{O}$ dans une lettre d'amour: "O's but the next door to $\mathrm{P}$, and his mistress may use her O with - with modesty" (IV.2.230-31). Plus élégamment, c'est un livre, "the two-leav'd book" qu'ouvre la maîtresse à ses amants dans la traduction de Marlowe des Amores d'Ovide (III.13.44) - métaphore novatrice, là où le latin dit plus vaguement "probra". Il y eut, entre lecture et jouissance, une longue lune de miel ${ }^{7}$. Mais tout autant que la lecture, l'écriture amoureuse est émotionnellement chargée. Aussi Bassanio, dans The Merchant of Venice, menace-t-il le jeune clerc de lui gâter sa plume (IV.1.237). La double signification du mot "sheet" permet à Beatrice, dans Much Ado about Nothing, d'écrire son nom et celui de Benedick sur la même feuille de papier et, en relisant la lettre, de trouver "Benedick and Beatrice between the sheet" (II.3.134-36). Semblablement, dans The Atheist's Tragedy, à Levidulcia qui lui promet d'écrire pour lui un mot ou deux, Sebastian réplique "A word or two, madam? That you do for me will not be contained in less than the compass of two sheets" (II.5.57-9). Les deux activités sont rapprochées par leur créativité ou leur fécondité communes; c'est une vitalité d'origine en partie érotique qu'insuffle au roi de France la jeune Helena, dont le toucher a le pouvoir, explique Lafew, "To give great Charlemain a pen in's hand / And write to her a love-line" (All's Well that Ends Well, II.1.74-7).

Mais l'amour a ses plaies comme l'écriture ses trahisons. Trop optimiste, Alonzo, dans The Changeling, n'y voit que des coquilles auxquelles il ne faut point accorder plus d'importance qu'au texte :

If lovers should mark everything a fault, Affection would be like an ill-set book Whose faults prove as big as half the volume. (II.1.109-11)

Cependant, le texte amoureux peut se corrompre, et provoquer les insertions que constituent les annotations marginales des commentateurs. C'est le reproche qu'adresse le duc de Guise à son épouse lorsqu'il découvre sa liaison avec Mugeroun dans The Massacre at Paris :

Or hath my love been so obscur'd in thee

That others needs to comment on my text?

$(15.25-6)$

Le texte authentique s'en trouve ainsi souillé, comme le livre de son cœur que présente le cardinal au roi d'Espagne dans The Noble Spanish Soldier de Thomas 


\section{LE LIVRE ET LA BLESSURE}

Dekker, "A booke here claspt up, which too late you open'd, / Now blotted by you with foule marginall notes" (II.1.178-79). La blancheur de la page est salie par une encre illicite. Dans The Couragious Turk de Thomas Goffe, accusant le contraste en substituant à la plume d'oie la plume de corbeau, Amurath défend les femmes contre les satiristes qui plongent "thir Ravens quill in Stygian Inke, / To blast such heavenly paper..." (147-50). Un contraste semblable, ironiquement amplifié par la couleur du protagoniste, sous-tend l'exclamation d'Othello, "Was this fair paper, this most goodly book, / Made to write 'whore' on ?" (IV.2.73-4). Penthea meurt, dans The Broken Heart, "Pure as are unwritten papers" (IV.3.145). Cependant, dans le cahier abîmé qu'évoque Othello, l'écriture parasite est aussi une tache, qui invalide un contrat. L'écriture a valeur d'engagement, de preuve ou de témoignage, qui semblent d'autant plus forts ou impérissables qu'elle s'inscrit dans l'être même.

C'est ce que dit Dromio d'Ephèse, dans le registre comique, en transformant sa propre peau en parchemin, et en imaginant que ne font qu'une la main qui frappe et la main qui écrit :

That you beat me at the mart I have your hand to show. If the skin were parchment, and the blows you gave were ink, Your own hand-writing would tell you what I think.

(III.1.12-14)

Dans la pastorale, le parchemin est fourni par l'écorce des arbres sur laquelle les amoureux entaillent la preuve de leur amour; cette inscription est un témoignage, comme le rappelle Orlando dans As You Like It :

O Rosalind, these trees shall be my books, And in their barks my thoughts I'll character, That every eye which in this forest looks, Shall see thy vertue witness'd everywhere.

(III.2.5-8)

A deux reprises, dans une remarque de Jaques (III.2.255-56) puis de Rosalind (III.2.350-52), une note de distanciation est apportée par le rappel de la blessure ainsi infligée à l'arbre. Dans la Rosalynde de Lodge, où l'on écrit beaucoup sur les arbres, semblable souci n'est jamais exprimé. En revanche, dans l'Orlando Furioso de Greene, lorsqu'il découvre les noms de Medor et d'Angelica gravés ensemble, Orlando s'indigne: "who wronged happy Nature so, / To spoyle these trees with this Angelica ?" (680-81). C'est que les noms entrelacés témoignent à ses yeux d'un amour consommé entre celle qu'il aime et son rival. Dans l'épisode correspondant de l'Arioste $(19,36 ; 23,103)$, cette écriture disait vrai, mais elle n'est ici qu'une fausse preuve, fabriquée par Sacripant dans le rôle d'un Iago. Quoi qu'il en soit, l'ironie sous-jacente au motif de l'amour entaillé dans l'écorce des arbres est suggérée dans le texte de Lodge aussi bien que dans celui de Greene. Dans Orlando Furioso, le valet de Sacripant, déguisé en berger pour mieux tromper Orlando en confirmant sa lecture des arbres, ressemble à "Paris (when Oenone lovd him well)" 
(576); plus clairement encore, dans la Rosalynde de Lodge, Aliena met en cause la fidélité de Rosador : "if I should ever proove as amorous as Oenone, I might finde as faithfull a Paris as your selfe ${ }^{8}$ ". En effet, depuis les Hérö̈des d'Ovide, les entailles de l'écorce donnent à lire un amour blessé; dans la cinquième épître, Enone écrit au berger qui l'a trahie en lui rappelant :

Mon nom demeure dans les hêtres que tu as entaillés; on y lit 'Enone' tracé par ton couteau, et à mesure que croissent les tiges, mon nom croît d'autant ${ }^{9}$.

Et lorsque dans le vers suivant, elle demande aux jeunes arbres de croître et de s'élancer "in titulos [...] meos" (V.24), unissant indissociablement les deux sens de titulus, l'inscription et le juste titre, elle trouve dans l'écrit l'essence du droit.

Or, ce qui devrait être une marque éternelle d'amour parce que gravée sur le vivant se métamorphose dans Titus Andronicus en éternité de la douleur écrite sur les cadavres; Aaron se vante :

Oft have I digg'd up dead men from their graves, And set them upright at their dear friends' door, Even when their sorrows almost was forgot, And on their skins, as on the bark of trees, Have with my knife carved in Roman letters, 'Let not your sorrow die, though I am dead'

(V.1.135-40)

Au retournement de la promesse amoureuse en inscription macabre s'ajoutent de multiples ironies. Le choix des lettres romaines par le barbare goth est symptomatique dans une pièce qui, selon la thèse de Grace Starry West, explore la dissociation tragique entre livre et civilisation ${ }^{10}$. A cela s'ajoute l'utilisation perverse de cadavres pour servir à la fois de livre de mémoire et de livre d'amitié. Mais c'est plus souvent sur les corps toujours vivants que les meurtriers aiment à écrire de la pointe de leur lame ${ }^{11}$. Dans The Noble Spanish Soldier le cardinal conseille à Medina "not to text downe / These wrongs in red lines" (IV.2.208-09). Amintor menace Evadne : "on thy flesh / I'11 print a thousand wounds" (The Maid's Tragedy, II.1.282-83). Plus vindicatif encore, Montsoreau, dans Bussy d'Ambois, oblige son épouse à écrire une lettre qui attirera son amant dans un guet-apens; comme elle s'y refuse, il la frappe à plusieurs reprises de son poignard :

Till thou writ'st, I'll write in wounds (my wrong's fit characters) Thy right of sufferance. Write! 
La fusion des deux sens de titulus qu'avait opérée (Enone est ici inversée dans l'homophonie right / write, qui, de façon sardonique, légalise la souffrance dans l'écriture. Méthodique, le traître Eleazar, dans Lust's Dominion, installe une chambre de torture qu'il appelle sa bibliothèque; chaque prisonnier y est un livre de choix (V.3.65-7), et le plus précieux a droit à de meilleures chaînes "For fear the Readers steal the art away" (V.3.68-70); dans cette bibliothèque, Eleazar s'apprête à dérouler "The volume of all wounds, that wound from me" (V.3.59). En intensifiant l'identification d'une série de blessures au déroulement d'un volume, le jeu de mots fait parader l'acte destructeur dans les habits volés de la créativité littéraire.

Parallèlement, l'écriture de la haine se superpose à celle de l'amour, dont l'encre est aussi le sang; lorsque dans Bussy d'Ambois Montsoreau écrit ses griefs dans le corps de son épouse, il parodie les lettres d'amour que Barrisor (II.2.155, 243) puis Tamyra (V.1.176) écrivent de leur propre sang. Le sang, "encre des amants", ricane Montsoreau; "témoin sacré", réplique Bussy (V.3.99-100). L'encre de l'amour est également celle de la vengeance, "this bloody writ", que fait tomber Bel-Imperia aux pieds de Hieronimo dans The Spanish Tragedy (III.2.26) ou les "bloody lines" que montre Titus à Tamora, en jurant que "what is written shall be executed" (Titus Andronicus, V.2.14-5). C'est aussi l'encre du pacte avec le diable (Doctor Faustus, II.1). La permanence et la solennité d'un contrat sont également figurées par l'inscription à vif dans le cœur. Tandis qu'Absalon porte la mort d'Ammon profondément gravée en son cœur (David and Bethsabe, 738-39), la duchesse de Guise, écrivant à son amant, voudrait pouvoir le faire à l'aide d'une plume arrachée à l'aile de Cupidon "That it might print these lines within his heart" (The Massacre at Paris, 15.10-12). "I'le write / This love within the tables of my heart" promet Biancha à Fernando dans Love's Sacrifice (éd. 1633, sig. F $2 v^{\circ}$ ). Celui-ci avait affirmé qu'arraché de son cadavre, son cœur donnerait à lire "Biancha's name carv'd out in bloody lines" (sig. $\mathrm{Fr}^{\circ}$ ); et solennellement, Biancha prononce, dans les mêmes termes, le serment réciproque (sig. F $3 \mathrm{r}^{\circ}$ ). Dans 'Tis Pity She's a Whore, Giovanni fait appel à la même image d'un sentiment profondément imprimé. Découvrant sa poitrine, et tendant à Annabella son épée, il s'écrie :

And here's my breast; strike home!

Rip up my bosom; there thou shalt behold

A heart in which is writ the truth I speak.

(I.2.209-11)

Ce dévoilement intime implique aussi une occultation que Soranzo, imitant Alsemero dans The Changeling (V.3.36-9), cherche à percer en voulant arracher le cœur d'Annabella pour y lire son secret (IV.3.53-4). Il en est prévenu par Giovanni, qui, brandissant le cœur de son amante à la pointe de son poignard, se dit "gilt in the blood / Of a fair sister" (V.6.69-70), transmuant ainsi, par le sang, la noirceur de l'inceste en glorieuses broderies d'or. Ce faisant, il amplifie la tentative d'Annabella, qui, voyant que sa conscience lui oppose des "depositions character'd in guilt" (V.1.10), s'approprie ces lettres dorées de la culpabilité en écrivant une mise en garde "double-lin'd with tears and blood" (V.1.34). Mais la noirceur de la faute a 
beau chercher à se rédimer dans l'or du sang, elle ne peut se constituer en texte signifiant; fasciné par la matérialité de l'écrit, Giovanni ne peut en lire le message :

\section{Tis her hand, \\ I know't; and 'tis all written in her blood. \\ She writes I know not what.}

Son effort désespéré pour donner à lire l'écriture du cœur est réduit à néant par la répulsion qu'inspire un objet sanguinolent désormais vide de signification.

C'est sur cette aporie que butte l'incessant désir du déchiffrement de l'être, constamment stimulé par la résistance au regard de "the heart's close book"(Edward III, II.1.305). Lady Macbeth reproche à son mari une trop grande lisibilité : "Your face, my Thane, is as a book, where men / May read strange matters" (I.5.61-2); mais Duncan, dans la scène précédente, venait de remarquer : "There's no art / To find the mind's construction in the face" (I.4.11-2). C'est peutêtre ce que découvre Hamlet lorsqu'il tente désespérement de déchiffrer le visage d'Ophelia :

He took me by the wrist, and held me hard.

Then goes he to the length of all his arm, And, with his other hand thus o'er his brow, He falls to such perusal of my face As he would draw it. Long stayed he so.

En vérité, comme le déplore Armostes dans The Broken Heart, la connaissance d'autrui reste tributaire de ses paroles car l'œil ne peut lire les pensées :

'tis the tongue informs the ears;

Our eyes can never pierce into the thoughts,

For they are lodg'd too inward.

Ce sentiment frustrant d'une intériorité opaque stimule le désir de pénétrer par le regard jusqu'aux profondeurs intimes de l'être. Dans The Conspiracy of Charles, Duke of Biron, le portrait de La Fin que brosse le roi retourne le personnage comme un gant de chevreau et porte l'intérieur à la surface ou l'obscur à la lumière :

Your majesty hath with the greatest life

Describ'd a wicked man, or rather thrust

Your arm down through him to his very feet

And pluck'd his inside out [...]

And those strange characters, writ in his face, 
Which at first sight were hard for me to read

The doctrine of your speech hath made so plain

That I run through them like my natural language.

(I.1.165-73)

Selon le duc de Savoie, le roi vient précisément d'opérer ce qu'Armostes dit impossible dans The Broken Heart, la transformation de l'oreille sans défense en regard pénétrant, "turn'd ears to eyes"(I.1.169). Dans The Raging Turke, refusant d'écouter plus avant les injures de son fils Selym, Bajazeth menace de substituer l'œil à l'oreille de manière à doubler cette transposition d'une torture :

Hold, hold thy venom'd tongue, if there be hid

More of this kind un-uttred, Ile rip up

Thy full-fraught bosome, and to save mine eare

Mine eyes shall overview what I'le not heare.

(2485-88)

Cette volonté de substituer l'écriture, puis la lecture à une perception auditive peu fiable est illustrée par certaines manipulations ou adaptations du mythe de Marsyas. L'aspect habituellement mis en vedette, chez des auteurs comme Chaucer (House of Fame, 1229-32) ou Watson (Hekatompathia, 15), dans la tradition de Plutarque ${ }^{12}$, est la compétition entre la flûte et la lyre. La musique est transformée, dans The Spanish Tragedy, en écriture; la poésie qu'adresse Balthazar à Bel-Imperia demeure impuissante, car, lui-même l'avoue,

The lines I send her are but harsh and ill,

Such as do drop from Pan and Marsyas' quill.

(II.1.15-6)

Il n'est donc guère surprenant que Hieronymo, après avoir fait de la création théâtrale l'instrument du meurtre, après s'être arraché la langue pour étouffer la parole, assassine le duc et se tue lui-même, non avec une épée ni un poignard mais, nouant indissociablement l'écriture et la mort, avec un canif destiné à aiguiser sa plume; c'est avec le même instrument que Doctor Faustus fait couler son sang pour écrire le pacte infernal dans la version anglaise du Faustbuch ${ }^{13}$, et c'est toujours le même accessoire de l'écrivain qu'utilise De Flores pour se donner la mort au terme de sa dernière mise en scène (The Changeling, V.3.174).

Une deuxième transposition s'opère dans The Tragedy of Hoffman, de Henry Chettle. Le protagoniste contemple avec fierté la victime qu'il vient d'écorcher :

If there live ere a surgeon that dare say

He could doe better: Ile play Mercury,

And like fond Marsias flea the Quacksalver.

(éd. 1631, sig. $\mathrm{C}_{2} \mathrm{v}^{\circ}$ ) 
Alors que dans The Spanish Tragedy le dieu de la musique s'effaçait devant celui de la création littéraire, ici, Apollon cède la place à Mercure, pour l'occasion dieu des chirurgiens; c'est remettre au premier plan l'acte d'écorcher, indissociable, chez Ovide, de l'exquise précision des notations visuelles :

Detectique patent nervi trepidaeque sine ulla

Pelle micant venae; salientia viscera possis

Et perlucentes numerare in pectore fibras.

(Métamorphoses, VI.389-91)

ses muscles, mis à nu, apparaissent au jour; un mouvement convulsif fait tressaillir ses veines, dépouillées de la peau; on pourrait compter ses viscères palpitants et les fibres que la lumière vient éclairer dans sa poitrine ${ }^{14 .}$

Ou, dans la traduction de Golding :

the sinewes lay discovered to the eye,

The quivering veynes without a skin lay beating nakedly.

The panting bowels in his bulke ye might have numbred well, And in his brest the shere small strings a man might easly tell.

(VI.495-98)

Les interprétations tardives du mythe de Marsyas trahissent un mélange de fascination et d'horreur devant la lecture anatomique. En net contraste avec Raphaël, dont la fresque lumineuse et sereine de la voûte de la Chambre de la Signature (1508) célèbre dans l'écorchement le triomphe de l'âme sur la prison corporelle ${ }^{15}$, le Titien met en scène le malaise (Le Châtiment de Marsyas, 1570, Kromeriz); l'obscurité trouble dans laquelle se déroule le supplice est dramatiquement trouée par quelques flèches de lumière qui viennent cruellement frapper le corps sans défense, nerveusement tendu, alors que les scalpels commencent leur œuvre. Comme le tableau du Titien, la tragédie élisabéthaine semble inverser l'assurance des planches anatomiques de Vésale et de ses imitateurs où, pour dévoiler complaisamment ses entrailles, l'écorché triomphant et sûr de lui, écarte les deux pans de sa peau comme s'il ouvrait les pages d'un livre. S'arrogeant la prérogative divine de sonder les reins et les cœurs, le regard inquisiteur cherche à déchiffrer les secrets de l'être dans la dissection anatomique. C'est pourquoi, en butte au mystère de la nature humaine, le roi Lear souhaite voir disséquer Regan (III.6.76); dans The Atheist's Tragedy, pour trouver le secret de sa fortitude, d'Amville demande qu'on lui livre le cadavre de Charlemont "For an anatomy" (V.2.143); dans Bussy d'Ambois, Montsoreau cherche à s'emparer de son rival

That I may hang him, and then cut him down,

Then cut him up, and with my soul's beams search

The cranks and caverns of his brain. 
De façon inquiétante, torture et connaissance se superposent ici, dans ce que Piero Camporesi nomme "l'atroce désir de savoir 16". A l'époque où Phineas Fletcher explore les circonvolutions internes de "The Purple Island" et, comme John Day, voit dans les merveilles anatomiques une preuve de l'existence de Dieu ${ }^{17}$, la tentative de rendre l'être lisible jusqu'aux tréfonds joue peut-être un rôle semblable à celui de certaines représentations du livre, où Marie-Madeleine Martinet a décelé une assertion de la science accompagnée d'une image de sa vanité ${ }^{18}$.

Pour autant que l'on puisse tenter de déterminer une ligne générale sans déformer, dénaturer ou simplifier à l'excès, il semblerait que s'organise, autour de l'image du livre humain ou du corps écrit et déchiffré, un système qui peut ne pas être dépourvu de cohérence. Par contraste avec l'instabilité de la plupart des textes, l'écriture qui s'imprime dans le vivant est souvent perçue comme indélébile, et c'est pourquoi le livre humain constitue le plus sacré des contrats. Aussi, dans The Spanish Soldier, le Roi brûle-t-il en vain le parchemin sur lequel est consigné l'engagement qui le lie à Enaelia, car celui-ci, comme le remarque le cardinal, qui s'inspire sans doute de la deuxième Epître aux Corinthiens (3.3), est profondément gravé dans le cœur de tous les témoins (II.1.207-11). Mais alors que la vie intérieure semble d'abord venir naturellement s'inscrire à la surface du corps comme en un livre facile à lire, celui-ci bientôt s'efface, se noircit, se déchire ou se ferme. Il faut alors, pour lire l'homme, l'ouvrir de force jusqu'au plus profond. Le contrat toujours écrit, métaphoriquement ou littéralement, de son propre sang, trouve à la fois sa caricature et sa négation dans l'écriture du poignard, mais aussi dans la volonté d'appropriation qui incite le regard à percer jusqu'au cœur. Si la connaissance, sur tous les plans, est une jouissance, elle est aussi indissociable de la douleur, et le désir de connaître, porté jusqu'à son extrême limite, finit par s'identifier à la volonté de détruire. Après s'être substitué au livre de l'autorité scolastique, le livre de l'expérience et de la vie menace de se retourner en livre du meurtre ou du supplice. Les superpositions et les inversions produites par les plis de l'imaginaire répondent à de multiples tensions entre acte fondateur et acte destructeur, entre l'enthousiasme et le doute, l'extase et le tourment. Un autre retournement pourrait être suggéré par une remarque de Marc Le Bot, selon qui

Affronter l'autre et soi-autre est un jouir et une douleur. Un corps, symboliquement, s'éventre: il affronte son intérieur à la présence réelle du dehors; et, symboliquement, il meurt dans le mouvement d'écriture qui fait, de son intérieur, un dehors. Le corps qui, dans l'écriture, affronte sa propre altérité à soi et l'altérité de tout autre, s'ouvre et fait plaie. Ecrire serait panser les plaies ouvertes par l'écriture $e^{19}$

Mais c'est d'une autre façon que la poétique de la Renaissance envisage la transmutation du corps qui s'écrit. Dans la poésie amoureuse, la dissolution de la chair dans l'écrit permet au poète de mourir à lui-même pour renaître dans l'écriture; il s'agit d'une fiction commune ${ }^{20}$, qui trouve peut-être l'une de ses sources lointaines dans 
l'appel de Dante à Apollon au seuil du Paradis, "Entra nel petto mio, e spira tue / si come quando Marsïa traesti / de la vagina de le membra sue" (I, 19-21) - "Entre dans ma poitrine, et souffle, toi, / comme quand tu as tiré Marsyas / hors de la gaine de ses membres ${ }^{21}$ ". Sur un plan différent, Spenser, comme bien d'autres auteurs de sonnets amoureux, prétend transformer la douleur en extase poétique par des vers "written with teares in harts close bleeding book" (Amoretti, I, 8). Les métaphores du livre humain dans le théâtre de la Renaissance anglaise répondraient aussi à une tension fondamentale (peut-être homologue de celle qui existe entre le Marsyas de Raphaël et celui du Titien), entre l'exaltante transfiguration du corps blessé en corps qui s'écrit et la misérable réduction du corps qui cherche à s'écrire en corps écrit, c'est-à-dire, dans l'un des sens d'imprimere, entaillé, voire écorché.

Yves PEYRÉ

Université de Toulouse-Le-Mirail

1 Goethe, Maximen und Reflexionen, cité par Ernst Robert Curtius, La Littérature européenne et le moyen-âge latin, P.U.F., Paris, 1956 (Francke, Berne, 1948), pp. 369-70.

2 Piero Camporesi, L'Officine des sens : Une anthropologie baroque, Hachette, Paris, 1989 (Garzanti, Rome, 1985), p. 71.

3 L'idée remonte à Pindare et aux tragédiens grecs : Curtius, p. 371.

4 Aussi Menenius tente-t-il de se substituer au livre que Coriolanus tient fermé : "I have been / The book of his good acts" (V.2.14-5). L'occultation sur le forum est le revers de l'exhibitionnisme qui, sur le champ de bataille, le dévoile aussi radicalement que Marsyas l'écorché. L'homologie Martius / Marsyas est suggérée par Poisson, Shakespeare Quarterly 15 (1964), p. 449 et par Bate, Shakespeare and Ovid, Clarendon Press, Oxford, 1993, p. 202 n. 48. Sur les relations entre Marsyas, l'écriture et la lecture, voir infra.

$5 \quad$ Voir aussi Yves Peyré, "Les mythes de la création littéraire dans la poésie amoureuse élisabéthaine", Les Mythes poétiques au temps de la Renaissance, éd. M.-T. Jones-Davies, Touzot, Paris, 1985, pp. 196, 199.

6 Voir aussi Marston, The Insatiate Countess, II.2.30-5.

$7 \quad$ Curtius, p. 386 n. 1. 


\section{LE LIVRE ET LA BLESSURE}

8 Lodge, Rosalynde, texte procuré par Furness dans l'éd. New Variorum de As You Like It, Philadelphie, 1892, pp. 316-87, p. 351.

9 Ed. Henri Bornecque, tr. Marcel Prévost, Les Belles Lettres, Paris, 1965 (1928), V.21-3.

10 Grace Starry West, "Going by the Book: Classical Allusions in Shakespeare's Titus Andronicus", Studies in Philology, 79 (1982), pp. 62-77.

11 Dans la tradition médiévale les blessures qu'infligent les bourreaux aux martyrs sont "une écriture pourpre" : Curtius, pp. 380, 385.

12 Voir Edgar Wind, "The Flaying of Marsyas", Pagan Mysteries in the Renaissance, Faber and Faber, Londres, 1968 (1958), pp. 171-76.

13 "he tooke a small penknife, and prickt a vaine in his left hand", The Historie of the Damnable Life, and Deserved Death of Doctor Iohn Faustus, Thomas Orwin, Londres, 1592, p. 7.

14 Tr. Georges Lafaye, Les Belles Lettres, Paris, 1970 (1928).

15 Outre Wind déjà cité, voir André Chastel, Art et humanisme à Florence au temps de Laurent le Magnifique, P.U.F., Paris, 1961 (1959), pp. 48-54.

16 Camporesi, L'Officine des sens, p. 92.

17 The Travailes of the Three English Brothers, dans The Works of John Day, éd. Robin Jeffs, Holland Pr., Londres, 1963, p. 327.

18 Marie-Madeleine Martinet, "Le livre dans les tableaux de la Renaissance : Perspective directe ou perspective inversée ?", L'Europe de la Renaissance. Cultures et civilisations, Mélanges offerts à Marie-Thérèse Jones-Davies, Touzot, Paris, 1988, pp. 103-08.

19 Marc Le Bot, "Le corps écrit" in Corps écrit, 18 (L'Allégorie), PUF, Paris, 1986, p. 158.

20 Voir Peyré, "Les mythes de la création littéraire", pp. 197-99.

21 Tr. Jacqueline Risset, Flammarion, Paris, 1990. 